\title{
They are looking... why not interacting? Understanding Interaction Around the Public Display of Community Sourced Videos
}

\author{
João Casal, Rui José \\ Centro Algoritmi, Universidade do Minho, Guimarães, Portugal \\ \{joaocasal,rui\}@dsi.uminho.pt
}

\begin{abstract}
In this paper, we study the extent to which the presentation of pedagogical videos on a public display at a communal space of the school is able to promote engagement around those videos. The videos were produced by students from the school itself. Using a mobile application, students could rate, create comments or simply bookmark videos. The evaluation of the platform is made through logs analysis, direct observation and a collective interview with end-users. The results show that even though the videos were able to attract many students to the display, there were not many of them that actually used the application to interact with content. In the final discussion, we explore some of the reasons that may justify this behavior and also the extent to which these videos have managed to foster students' curiosity towards their topics.
\end{abstract}

\section{Introduction}

Public displays can play an important role as an enabling technology for Ambient Intelligence, and particularly as an important medium for informal learning. Their ability to break content bubbles and promote serendipitous encounters with pedagogical topics can help to foster curiosity and discussion around those topics. Our work is part of an on-going EU funded research project, called JuxtaLearn, which aims to promote students' curiosity in science and technology through creative filmmaking, collaborative editing activities and content sharing.

In this work, we aim to study the extent to which the presentation of pedagogical videos on a public display at a communal space of the school is able to promote engagement around those videos. This work follows on a participatory design process where teachers were invited to contribute to the identification of the properties of the display systems that would be deployed [1]. This process has produced an early prototype and also several insights into multiple institutional and ethical concerns associated with this presentation of information in the public space of the school [2]. The involvement of the teachers was also important to bring these key stakeholders into the project, opening the door to a greater appropriation and acceptance of the technology. We also promoted two activities with students that aimed to create videos about a diverse set of pedagogical topics. This way, we were able to use videos that 
had been created at the school itself, considerably raising the identification of viewers with the exhibited content. The videos were presented on the public display and students could use a mobile application to rate, create comments or simply bookmark them.

Our study aimed to understand the nature of the interactions, both implicit and explicit, generated by the presentation of the videos. To support the evaluation, we used a broad range of qualitative and quantitative techniques, such as logs analysis, direct observation and a collective interview with end-users. The results show that even though the videos were able to attract many students to the display, there were not many of them that actually used the application to interact with content. In the final discussion, we explore some of the reasons that may justify this behavior and also the extent to which these videos have managed to foster the curiosity of students regarding the represented topics.

\section{Related work}

Interaction with public displays is mostly expected to occur as part of a public setting where many people may be present, typically carrying out multiple activities and having their own goals and context. Therefore, for interaction to occur, the display must be able to attract and manage people's attention. However, engaging users with interactive public displays is known to be a challenging task. Brignull and Rogers, reported that "a major problem that has been observed with this new form of public interaction is the resistance by the public to participate" [3]. Kukka et al. studied how this barrier to interaction, what they call the 'first click' problem, can be overcome [4]. Previous research has also identified the display blindness effect [5], where people look at the display, but do not see its content. Based on previous experiences that created the expectation that content is not relevant, people just learn to filter it. Müller et al. pointed out that the majority of users only look at the displays if they have the expectation of seeing relevant content [5]. The fear of looking silly while interacting with the display, especially in gestural interfaces, has also been pointed out as another barrier to interaction [3]. Müller et al. also explore the issue of noticing the display interactivity as other barrier for interaction [6].

To better understand these issues, a number of audience behavior frameworks have been proposed to represent the various phases of engagement with public displays and the transitions that occur between those phases. Michelis and Müller describe the Audience Funnel [7] as a framework that focuses on observable audience behavior. This framework is derived on observations from the Magical Mirrors installation, which consists of 4 displays showing a mirror image of the environment in front of them, overlaid with optical effects that react to the gestures of the audience as recognized by a simple motion detection system based on a single camera. The Proxemic Peddler [8] is a prototype public display system that proposes a continuous proxemics interaction framework that takes into account users' identity, distance, and orientation relative to the public display. These multiple audience models have been created for specific purposes, and despite their common patterns they are also highly dependent on particular assumptions about the display setting and usage context. Our 
work differs in the use of video as the primary media, and in the social context, which may be described as a learning community where most people know each other and in many cases even know the people acting on the videos. Moreover, our methodology of data recovery, categorizing the behavior of mobile users on the ubiquitous environment and attempting to link the in-situ observations with the system logs, also differs from previous work.

\section{The Public Display System}

The public display used for this study includes a display application that renders the videos published by students and shows some additional information about them. In the space between videos, the audience is informed about which video was shown last and which are to be shown next. The application also displays metadata associated with the videos like title, author, rating and number of votes. Using our mobile application, users can also access this same information on their mobile devices. The mobile application shows a content stream with information about the recently presented videos, giving users easy access to rate, comment or simply access the video on YouTube. The rate feature allows users to classify the videos. The comment feature has the intent of allowing users to let authors know what they think about their creation. The third feature, know more, leads the user to the YouTube page of the video, which allows the personal viewing of the content or access related videos about the same issue. The application on the display frequently shows information about how to download and use the mobile application, incentivizing people to it. In addition to the videos, at regular intervals, the display system also runs other applications that show school information like news or photos of events.

To seed the system with locally relevant videos, we promoted a pedagogical video competition where students created a number of pedagogical videos across different scientific areas. The goal was to overcome the display blindness effect [5] by offering users content that they could more easily identify with and thus perceive as more relevant. To allow users to notice interactivity [6], we created informative digital posters that were being exhibited on the display regularly. The posters have also been posted on the institutional Facebook of the school. In order to raise the interaction with the system, one of the prizes of the video competition was awarded to the most interacted video. This had the goal of fostering the interaction of the participants and also to invite these users to ask their colleagues to interact, promoting a larger adoption of the system. Fig. 1 represents the physical layout of the communal room where the display was located.

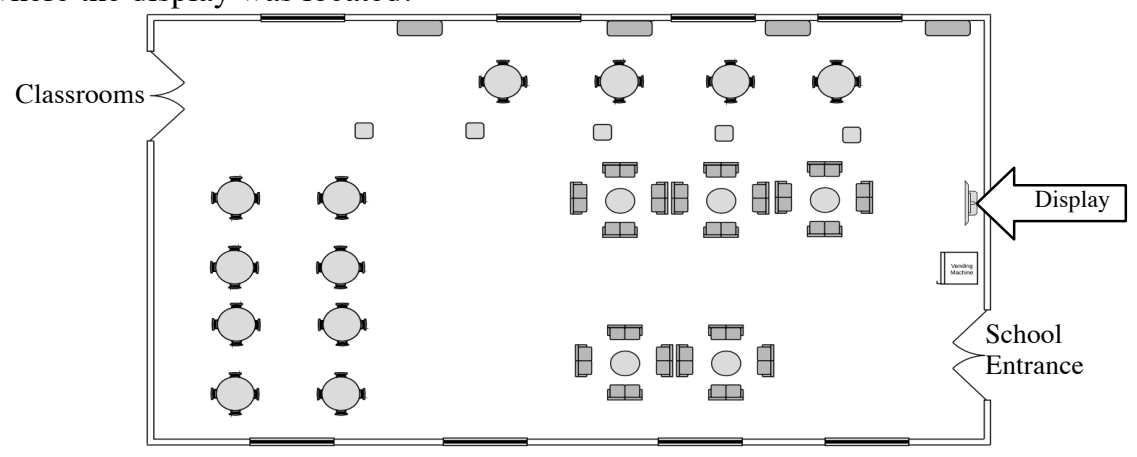

Fig. 1. Public display installation on school students' hall 
The location was selected as the place that would be able to capture students' attention, as this is a space where they hang around during breaks. The room is also a place that most students need to walk through as they go to or return from classes.

\section{Research Methodology}

The research methodology was based on a combination of three techniques: direct observations, analysis of the data recorded by the system and a collective interview to end-users. Direct observations were used to record users' behavior towards the system even before they start using the mobile application. However, to better understand the flow between these two distinct phases of interaction, we made the observations in a way that could later be associated with specific system events generated by that same user on the mobile application. To support this type of registration, we created an electronic form where we could register the observed behaviors along with an automatically generated timestamp. This allowed the researcher to be focused on the audience behavior, while at the same time generating the information that would latter allow these observations to be matched against specific system actions. In total, we conducted 4 hours and 35 minutes of observations in two distinct occasions. The analysis of the system logs was used to complement the direct observation with a concrete indication of the actions performed by users. The final group interview provided important insight on the reasons behind some of the observed behaviors. This interview involved three participants of the 12th grade (one male and two female) and lasted 45 minutes. This session was based on a semi-structured format of an informal conversation guided by key points.

\section{Results}

Figure 2 summarizes the key patterns of interaction identified during the observations as well as the frequency of occurrence of each.

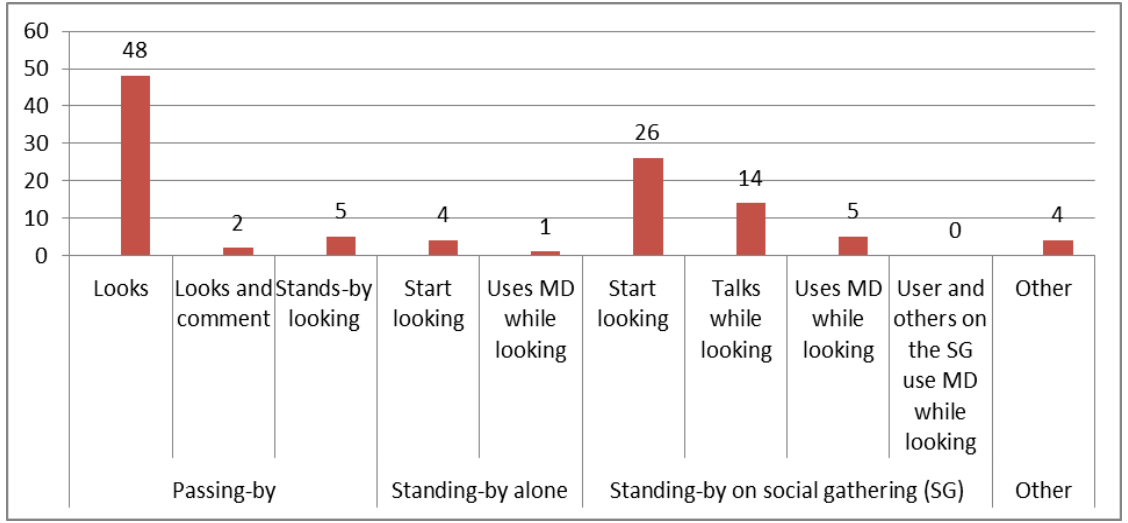

Fig. 2. Engagement events across different situational contexts 
Interaction with the public display starts with a simple glance, but then it can follow different interaction patterns according to the user context. It is important to mention that, in order to measure attention, the moment users stopped looking at the display were also annotated but, as that is redundant information for the analysis of this graph (because eventually all stop looking), it has not been represented.

We can also verify in Fig. 3 how the observed behaviors were different between the break time, when the room was completely crowded, and class time, when only occasional users were passing-by.

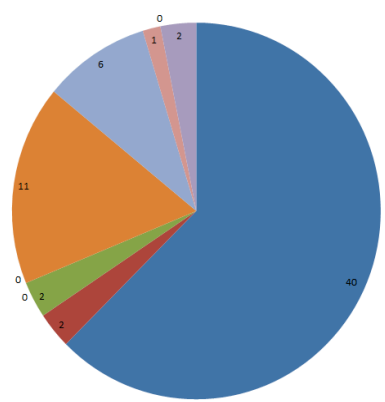

(A)

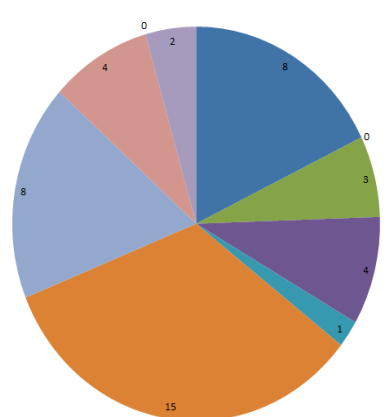

(B)

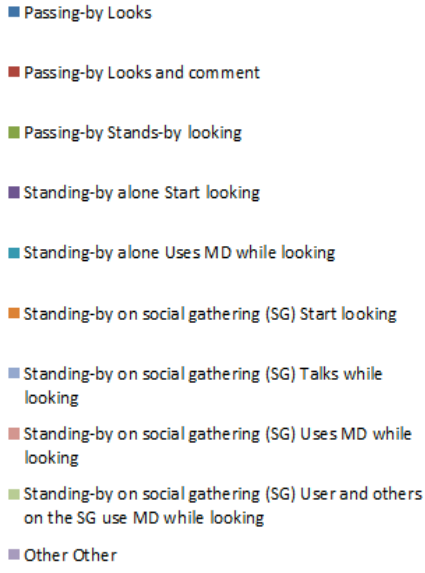

Fig. 3. Events observed during: (A) breaks; (B) classes

During the breaks, students are more involved in direct social interaction and not many of them pay attention to the display. However, since the room is crowded, the absolute number of engagement events is higher during these break periods. Table 1 shows that despite the existence of fewer events outside the breaks, they are more likely to be extended interactions, meaning that this is a period of greater availability.

Table 1 - Engagement events during breaks and classes

\begin{tabular}{|r|c|c|}
\cline { 2 - 3 } \multicolumn{1}{c|}{} & Break & Classes \\
\hline Number of observed events & 54 & 28 \\
\hline Average time engaged with the system & $1 \mathrm{~m} 13 \mathrm{~s}$ & $4 \mathrm{~m} 57 \mathrm{~s}$ \\
\hline Total time engaged with the system & $1 \mathrm{~h} 0 \mathrm{~m} 29 \mathrm{~s}$ & $1 \mathrm{~h} 54 \mathrm{~m} 01 \mathrm{~s}$ \\
\hline
\end{tabular}

Regarding the logs recorded on the system, the following results were obtained on approximately a month of use:

- 20 distinct users signed up (19 of which interacted with videos)

- 94 interactions with videos were registered;

- 2 distinct users wanted to know more about videos;

- In 9 videos, users followed the YouTube link in order to see them again or to watch related videos. 
Table 2 lists the interactions per type of production or type of content, giving insights about which are the video performances that foster more curiosity.

Table 2 - Number of interactions per type of video performance

\begin{tabular}{|c|c|c|}
\hline Type of production & \# of interactions & $\%$ of interactions \\
\hline Students performing & 59 & 62,8 \\
\hline Scenes shot on own city & 13 & 13,8 \\
\hline Content presentation & 9 & 9,6 \\
\hline Based on web resources (ex.: personas talking) & 5 & 5,3 \\
\hline Video tutorial alike & 3 & 3,2 \\
\hline Other (ex.: video contest advertisement) & 5 & 5,3 \\
\hline $\begin{array}{ll} & \text { Total }\end{array}$ & 94 & 100 \\
\hline
\end{tabular}

The group interview was intended to get a qualitative assessment of the deployment and we will now present the main results of this part of the study:

Current awareness and involvement. Every participant had heard about the video contest, knew about the existence of the display and has watched videos on it. However, no participant knew that the display is interactive ("the display don't seem interactive... how do I touch it?").

Motivations for use. As main motivations for using the ubiquitous system, participants stated that: (1) were curious to see the videos produced by persons they know; (2) motivation to watch or interact depends on the content ("if the video is from my area of interest I will more likely watch it" and "I usually look at the display because I know that my video is being exhibited there").

Interaction barriers. Students pointed out that: (1) "the display seems just a regular TV... we did not knew we could interact"; (2) "repetition of videos is boring"; (3) "the moment that persons are on that place is for relax not for learning"; (4) "display is too small for the size of the room; (5) "on my previous school existed TVs always displaying stuff and people ignored them"; (6) "I don't have a smartphone, so I cannot interact... however, I'm aware that in my class almost everyone has"; (7) Publicity, too much information, application errors or not understanding how to use are factors of disengagement; (8) "Features like rate are meant to be used once or twice, not to engage users on a system".

Interaction through personal mobile devices. Regarding the interaction mechanism implemented, users stated that: (1) "I was expecting that the display was touch"; (2) "it is a good bet. Nowadays everything can be done through smartphone. But it should allow other form of interaction for the persons that do not have smartphone."; (3) "it is better than touch displays if the features need personal authentication"; (4) "it is worse than touch displays in terms of catching users attention, because if I saw persons touching a display I would go there see what it was"; (5) "has the advantage of allowing multiple interactions simultaneously". 
Promotion of awareness towards interaction with the system. In order to promote awareness regarding how to interact with the system students suggested to have a physical poster near the display informing that it is interactive ("it is better than posters on the display because this information is always there, not only at some moments").

Video on interactive public displays versus video on YouTube. This question raised the following points of view: (1) "on YouTube it is possible to see the video without concerns about the rest of the audience"; (2) "it would be interesting to push videos to the public display for viewing with colleagues"; (3) "public displays make students see videos that they would not search for... and they may like it"; (4) "YouTube is meant for individual use and a video application on public displays is interesting for using on a social gathering context".

\section{Discussion and conclusion}

A clear finding from these results is the high number of users that pay attention to the display, possibly even commenting about its content with nearby colleagues, but never reach the point of interacting through their mobile devices. In regard to the high interest raised by the videos, the familiarity with the people and the scenes represented in many of those videos seem to have been a fundamental element to create this attention. When passing-by, students would often stop when they recognized a colleague acting on a video.

For the many videos creators, there was also the expectation of seeing their own videos being exhibited. These results seem to confirm that the public presentation of the videos can be a motivating element to the creation of those videos by students and also foster curiosity around those videos. Further research is needed to study the pedagogical relevance of this curiosity towards pedagogical content.

As for the reason why this high attention did not translate into high levels of mobile interaction, we essentially identified that many students never realized that there was this possibility. Despite the intensive communication effort and the video competition prize that was attributed to the video with more interactions, many students never understood they could interact using their mobile phone. This fact, together with students observations like "the display does not seem interactive... how do I touch it?" and "on my previous school existed TVs always displaying stuff and people ignored them" led us to conclude that previous knowledge of users regarding the interaction with public displays affects the adoption of novel forms of interaction. Students were expecting that if the display was interactive it was a touch display, and having that as a fact ignored the disclosed information.

As future work, we will study novel ways to promote interaction associated with the videos that raise curiosity. This essentially means increasing the conversion rate from viewers to users that act on display media. 
Acknowledgments. The research leading to these results has received funding from the European Community's Seventh Framework Programme (FP7/2007-2013) under grant agreement no. 317964 JUXTALEARN. We would like to sincerely thank school Escola Secundária de Alberto Sampaio (Portugal) for their collaboration on the technology deployment, on the promotion of the video competition and for the authorization to perform this research on their premises.

\section{References}

1. Otero, N., Alissandrakis, A., Müller, M., Milrad, M., Lencastre, J.A., Casal, J., José R.: Promoting secondary school learners' curiosity towards science through digital public displays. Proceedings of International Conference on Making Sense of Converging Media - AcademicMindTrek '13. pp. 204-210. ACM Press (2013).

2. Lencastre, J.A., Coutinho, C., Casal, J., José, R.: Pedagogical and Organizational Concerns for the Deployment of Interactive Public Displays at Schools. New Perspectives in Information Systems and Technologies. pp. 429-438 (2014).

3. Brignull, H., Rogers, Y.: Enticing People to Interact with Large Public Displays in Public Spaces. International Conference on Human-Computer Interaction INTERACT 2003. pp. 17-24 (2003).

4. Kukka, H., Oja, H., Kostakos, V., Gonçalves, J., Ojala, T.: What makes you click: exploring visual signals to entice interaction on public displays. Proceedings of the SIGCHI Conference on Human Factors in Computing Systems - CHI '13. ACM Press, New York, USA (2013).

5. Müller, J., Wilmsmann, D., Exeler, J., Buzeck, M., Schmidt, A., Jay, T., Krüger, A.: Display Blindness: The Effect of Expectations on Attention towards Digital Signage. Pervasive Computing: 7th International Conference. pp. 1-8. Springer Berlin Heidelberg, Nara, Japan (2009).

6. Müller, J., Walter, R., Bailly, G., Nischt, M., Alt, F.: Looking glass: A Field Study on Noticing Interactivity of a Shop Window. Proceedings of the 2012 ACM annual conference on Human Factors in Computing Systems - CHI '12. p. 297. ACM Press, New York, New York, USA (2012).

7. Michelis, D., Müller, J.: The Audience Funnel: Observations of Gesture Based Interaction With Multiple Large Displays in a City Center. Int. J. Hum. Comput. Interact. 27, 562 (2011).

8. Wang, M., Boring, S., Greenberg, S.: Proxemic peddler: a public advertising display that captures and preserves the attention of a passerby. Proceedings of the 2012 International Symposium on Pervasive Displays - PerDis '12. ACM Press, New York, New York, USA (2012). 